\title{
A near-infrared survey for Galactic Wolf-Rayet stars
}

\author{
N. L. Homeier ${ }^{1,2, \star}$, R. D. Blum ${ }^{3, \star}$, P. S. Conti ${ }^{4}$, and A. Damineli ${ }^{5, \star}$ \\ ${ }^{1}$ European Southern Observatory, Karl Schwarzschild Str. 2, Garching bei Muenchen, Germany \\ 2 University of Wisconsin-Madison, Astronomy Department, 475N Charter St., Madison, WI 53706, USA \\ 3 Cerro Tololo Interamerican Observatory, Casilla 603, La Serena, Chile \\ 4 JILA and APS Department, University of Colorado, Boulder, CO 8030, USA \\ 5 Instituto Astronomico y Geophísico, São Paulo, Brazil
}

Received 12 September 2002 / Accepted 22 October 2002

\begin{abstract}
Initial results, techniques, and rationale for a near-infrared survey of evolved emission-line stars towards the Galactic Center are presented. We use images taken through narrow-band emission-line and continuum filters to select candidates for spectroscopic follow-up. The filters are optimized for the detection of Wolf-Rayet stars and other objects that exhibit emissionlines in the $2 \mu \mathrm{m}$ region. Approximately three square degrees along the Galactic plane have been analyzed in seven narrow filters (four emission lines and three continuum). Four new Wolf-Rayet stars have been found and are the subject of a following paper.
\end{abstract}

Key words. stars: Wolf-Rayet - Galaxy: stellar content - Galaxy: center - infrared: stars

\section{Introduction}

Optical surveys within our Galaxy are severely hampered by dust obscuration; complete samples therefore must be obtained with longer wavelength studies. Here we describe a survey procedure for evolved massive stars in the Galactic plane at $K$ band wavelengths, where the extinction is 10 magnitudes lower than for traditional $V$-band surveys. Our scientific driver is the discovery of young stellar populations in our Galaxy through the detection of evolved massive stars. These stars have strong emission lines, which makes them relatively easy to detect using narrow-band filters.

Massive stars drive the evolution of galaxies through powerful stellar winds, large mass ejections, and explosive deaths. These mechanisms are the dominant source of energy input into the ISM; thus the structure and composition of the ISM in most galaxies is largely determined by the massive star population (Leitherer et al. 1992; Oey \& Clarke 1997; Heckman et al. 1998; Martin 1999; Oey et al. 2001; Heckman et al. 2001). Massive stars are also essential contributors to the chemical evolution of their host galaxy, ejecting material enriched in helium, carbon, and nitrogen during their lives, and depositing elements heavier than nitrogen in their final eruption as $\mathrm{SNe}$.

Send offprint requests to: N. Homeier,

e-mail: nhomeier@eso.org

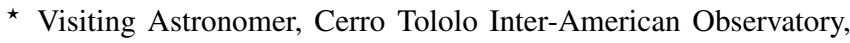
National Optical Astronomy Observatories, which are operated by the Association of Universities for Research in Astronomy, Inc., under cooperative agreement with the National Science Foundation.
As a massive star evolves, its spectrum becomes dominated by emission lines, arising either in a dense stellar wind, or in circumstellar material produced by mass loss. The presence and strength of individual lines are clues to the star's evolutionary state and atmospheric structure. Among evolved massive stars with such spectra, emission lines are most pronounced in Wolf-Rayet (WR) stars and in the Luminous Blue Variable (LBV, or S Dor variable) stage, where they shed large (1 to $10 M_{\odot}$ ) amounts of chemically enriched matter in a relatively small amount of time ( $10^{4}$ yrs) (Pasquali et al. 1997; Smith et al. 1998; Langer et al. 1999).

WR stars have lifetimes $<10 \mathrm{Myr}$, and thus are excellent tracers of recent star formation, and so also Galactic structure. They are also important in our quest to understand how star formation proceeds. For example, most of the previously known WRs are relatively isolated or in OB associations, but recent searches in the IR have found a plethora of these objects in compact clusters near the Galactic center (Blum et al. 1995; Krabbe et al. 1995; Nagata et al. 1995; Figer et al. 1999a; Blum et al. 2001).

\section{Life cycle of a massive star}

In the "Conti scenario", the evolution of a massive star is determined by the amount of mass loss it undergoes (Conti 1976; Maeder \& Conti 1994). This mass loss is driven by the combination of the star's intrinsic luminosity and opacity due to metal lines. The more luminous a star is, the greater the outward force, and the more metals present in the wind, the greater the opacity and hence the ability to drive a mass-losing stellar wind. Our picture of massive stellar evolution is rapidly 
Table 1. Filter description.

\begin{tabular}{cc}
\hline \hline Central $\lambda(\mu \mathrm{m})$ & $F W H M(\mu \mathrm{m})$ \\
\hline 2.032 & 0.010 \\
2.062 & 0.010 \\
2.077 & 0.015 \\
2.142 & 0.020 \\
2.161 & 0.022 \\
2.191 & 0.013 \\
2.248 & 0.024 \\
\hline
\end{tabular}

changing, as new models including rotation are developed (Maeder \& Meynet 2000; Meynet \& Maeder 2000). It now appears that rotation is second only to mass loss rate in its effect on massive stellar evolution, and it may even be more important at low metallicities (Maeder \& Meynet 2001).

The current qualitative overview of massive stellar evolution is as follows. For a star with an initial mass of $\geq 30 M_{\odot}$, mass loss and mixing on the main sequence deplete the hydrogen-rich envelope and reveal the equilibrium products of CNO-cycle hydrogen burning, creating a WN star. Additional mass loss and mixing reveals the products of He-burning, and the star becomes a WC star. The star will end its life as a Type Ib or Ic supernova. This evolution may or may not be punctuated by eruptions and episodes of large mass loss where the star is identified as an LBV.

\section{The need for NIR observations}

The ideal place to study massive star formation and evolution is our own Galaxy, where we can resolve objects on small linear scales and have some hope of complete samples. It would be of great interest to find all the WR stars in our galaxy to test stellar evolution theories at high metallicity, and to learn about environments of massive star formation. Another important benefit is the study of Galactic structure as traced by young star-forming regions.

Surveys for emission line stars in the Milky Way using the narrow band technique have been done before, but in the optical, where extinction by intervening dust is highly problematic (Shara et al. 1999). However, these surveys firmly establish WR stars as key tracers of star formation, both in the Milky Way and other galaxies (Conti 1991). By moving to the NIR, we can identify these objects in regions where heavy reddening renders them undetectable in the optical.

With a simple model for the distribution of Galactic WR stars, Shara et al. (1999) demonstrate the difficulty in conducting WR searches in the optical. Because of high extinction in the plane, the vast majority $(\sim 90 \%)$ of the Milky Way's massive stellar population is hidden from view by obscuring dust. They also showed how this could be overcome by moving to the infrared, indicating that the number of WR stars as a function of magnitude peaks at $K=13-14$. It is clear that NIR surveys within the plane of our Galaxy are essential to significantly enlarge the known population of WR stars.

\section{Survey description}

In Table 1 we present central wavelengths and FWHMs for our chosen set of $K$-band filters (Blum \& Damineli 1999a;
Table 2. Prominent lines in Wolf-Rayet $K$-band spectra.

\begin{tabular}{lll}
\hline \hline Central $\lambda(\mu \mathrm{m})$ & Transition & Type \\
\hline 2.0587 & He I $2 s-2 p$ & WN, WC \\
$2.0705 / 2.0796 / 2.0842$ & C IV $3 p-3 d$ & WC \\
$2.1126 / 2.1137$ & He I $4 s-3 p$ & WN, WC \\
$2.1038 / 2.1152 / 2.1155 / 2.1156$ & C III/N III $8-7$ & WC \\
2.1632 & He I $7-4$ & WN, WC \\
2.1652 & He II $14-8$ & WN, WC \\
2.166 & Br $\gamma$, H I $7-4$ & WN \\
2.189 & He II $10-7$ & WN, WC \\
2.2779 & C IV 15 -12 & WC \\
2.3178 & C IV 17 -13 & WC \\
2.3470 & He II $13-8$ & WN \\
\hline
\end{tabular}

Data is reproduced from Table 2 of Figer et al. (1997), see references therein. Wavelengths listed are vacuum wavelengths.

Homeier et al. 2002). Four filters are centered on the characteristic stellar wind emission lines of He I $2.06 \mu \mathrm{m}$, C IV $2.08 \mu \mathrm{m}$, $\mathrm{H}$ I Br $\gamma 2.166 \mu \mathrm{m}$, and He II $2.189 \mu \mathrm{m}$, and the additional three continuum filters are at $2.03 \mu \mathrm{m}, 2.14 \mu \mathrm{m}$, and $2.248 \mu \mathrm{m}$. Thus each line filter measurement has a continuum point to the red and blue. It is extremely important to have continuum points to the red and the blue of each line filter because of the variation in continuum slope caused by dust extinction.

In Table 2 we list the most prominent emission lines in WR spectra, and the filter response curves are overplotted in Figs. $1 \mathrm{a}$ and $1 \mathrm{~b}$ for unpublished $K$-band spectra of WN and WC stars kindly provided by P. Eenens. In both, the three continuum filters are overplotted as dot-dash lines, and the four line filters as dotted lines. This illustrates the sensitivity of the 2.17 and 2.19 filters to WN stars, whereas the 2.06 and 2.08 filters are most sensitive to WC stars.

\subsection{Observations and reductions}

In 1996 the survey was begun at the $1.5 \mathrm{~m}$ at the Cerro Tololo Interamerican Observatory, and continued in 1997, 1998, and 2000. For 1996, 97, and 98 we used the same telescope and instrument configuration: the Cerro Tololo Infrared Imager (CIRIM) with the $\mathrm{f} / 8$ mode, giving us a $5^{\prime} \times 5^{\prime}$ field of view and $1.16^{\prime \prime}$ per pixel. In 2000, we used the Ohio State Infrared Imaging Spectrometer in the $\mathrm{f} / 8$ mode for a $10^{\prime} \times 10^{\prime}$ field of view and $1.16^{\prime \prime}$ per pixel. Our images are taken in "strips" composed of 35-45 images. We offset 1/3 of a chip in RA (19961998 data) or Dec (2000 data), keeping the other coordinate constant. Thus each spot on the image strip is a composite of three exposures. Images have been obtained over $\sim 3$ degrees of longitude between \pm 0.5 near the Galactic Center. This is shown in Fig. 2, and overplotted on a $90 \mathrm{~cm}$ image of the Galactic Center region in Fig. 3. In 2000, we moved outward along the plane to $l=316$, at the edge of Centaurus looking towards the Scutum-Crux spiral arm. In this region we obtained $\sim 1$ degree of Galactic longitude between \pm 1.0 Galactic Latitude. This is shown in Fig. 4. A sample of our data is shown in Fig. 5, with a small rectangle indicating the approximate position on the $90 \mathrm{~cm}$ radio image in Fig. 3. 

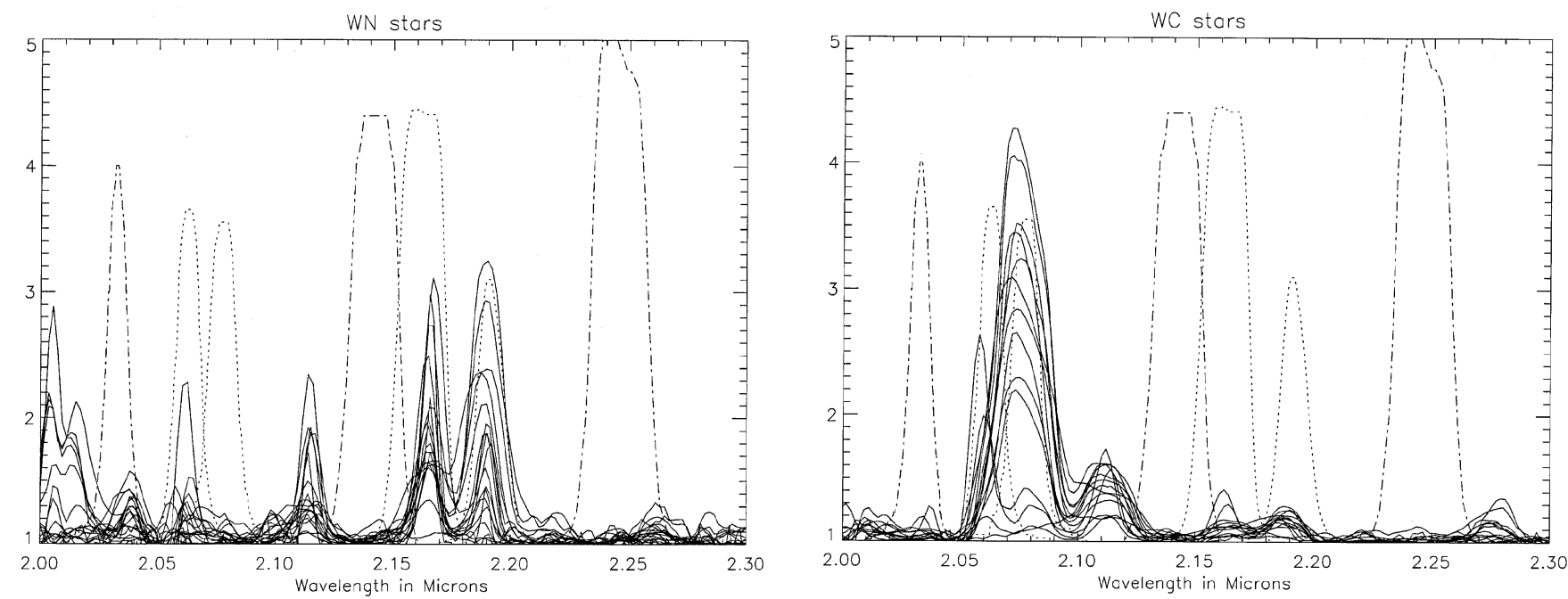

Fig. 1. A representative sample of WR stars with filter transmission curves overplotted, WN stars left panel and WC stars right panel. Dotted curves represent the line filters at 2.06, 2.08, 2.166, and 2.19 $\mu \mathrm{m}$. Dot-dashed lines represent the continuum filters at 2.03, 2.14, and 2.248 $\mu \mathrm{m}$. The He II filter transmission curve is difficult to see in the left panel due to the strong He II lines in the WN stars. WR spectra in both panels are kindly provided by P. Eenens.

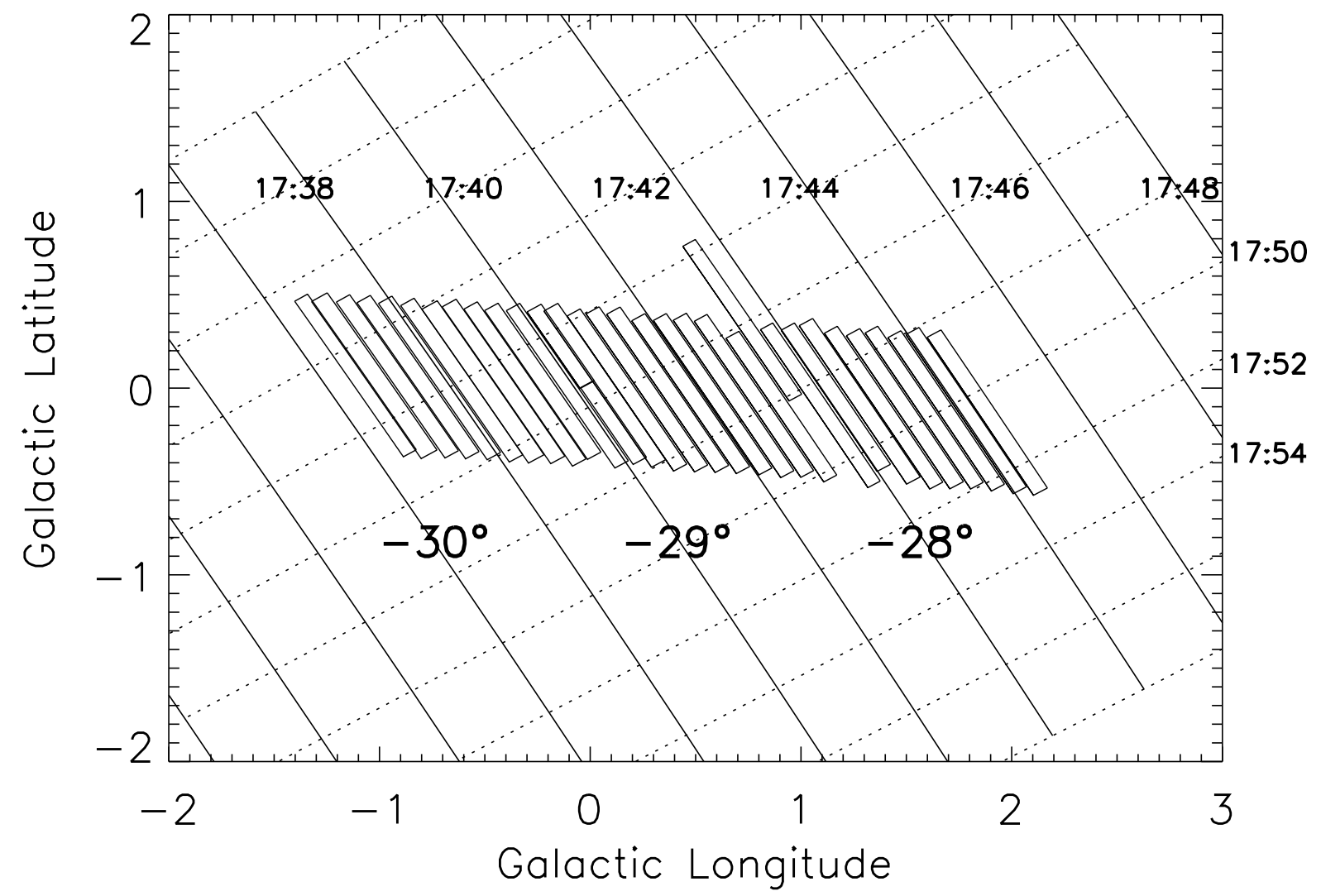

Fig. 2. Map of the observations from 1996, 1997, and 1998. The $x$ and $y$ axes are in Galactic coordinates. Lines of constant right ascension are overplotted in intervals of $2 \mathrm{~min}$ as dotted lines, and lines of constant declination are overplotted every half degree as solid lines.

Data reduction is performed with the CIRRED package of routines in IRAF $^{1}$, written specifically for CIRIM and OSIRIS ${ }^{2}$

\footnotetext{
${ }^{1}$ IRAF is distributed by the National Optical Astronomy Observatories.

2 OSIRIS is a collaborative project between the Ohio State University and CTIO. OSIRIS was developed through NSF grants AST 90-16112 and AST 92-18449.
}

reductions by R. D. Blum. The reduction steps are as follows. First, we trim the flat-field images if needed. For each filter there is a set of images taken with the lamps on and off. We take the median of each set of "on" and "off" frames to make a single "on" and "off" image. We subtract the "off" frame from the "on" frame to make a flat image, which is then normalized by the mean. A bad pixel mask is made by comparing the dome 


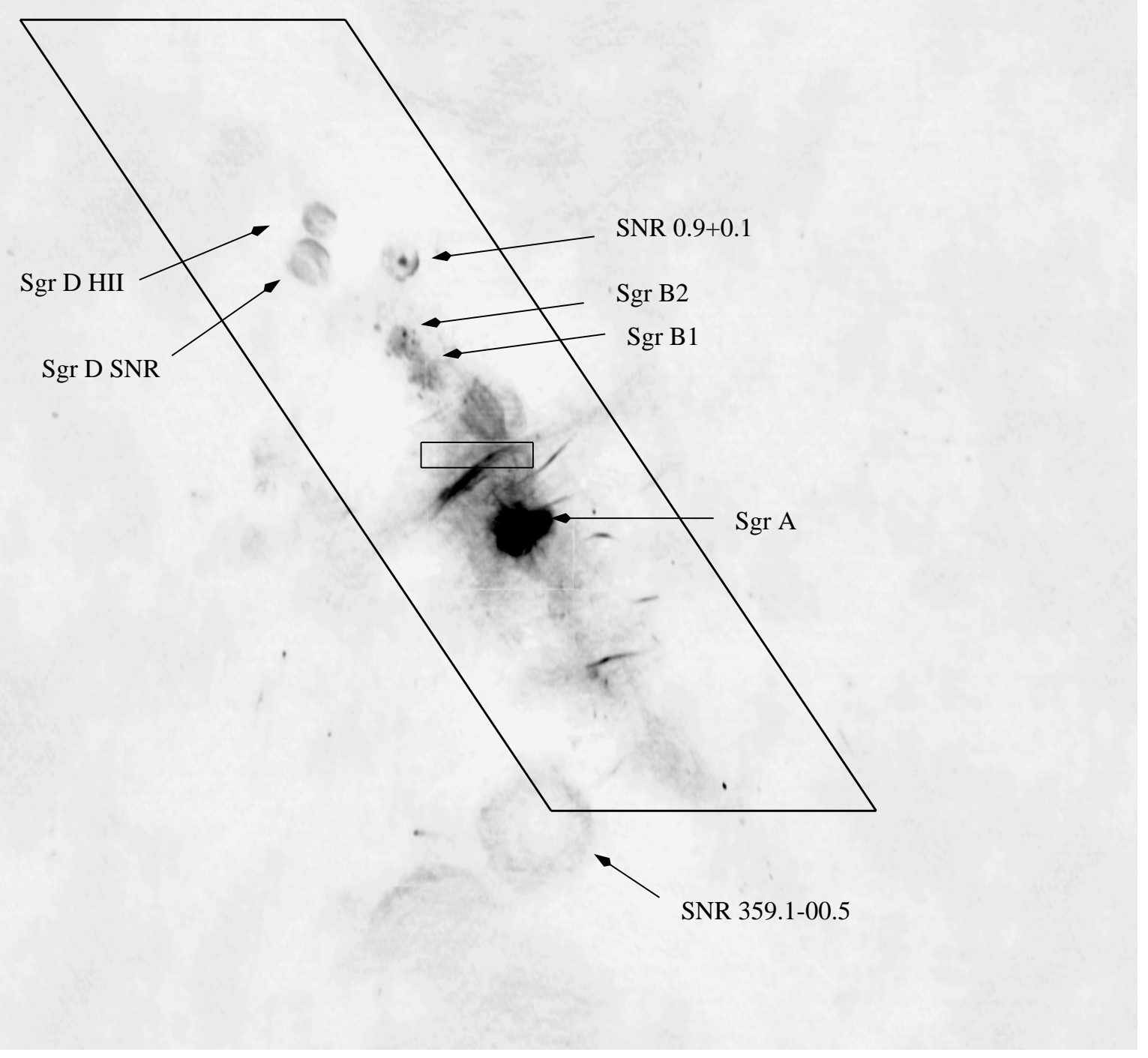

Fig. 3. The same data as in Fig. 2 overplotted on the $90 \mathrm{~cm}$ radio image of the Galactic Center region presented in LaRosa et al. 2000. Major features are labeled, and the small rectangle indicates the approximate position of Fig. 5 .

flats with the lights on and the lights off and using the histogram of pixel intensities to distinguish good and bad pixels. This bad pixel mask is then used to correct the flat field images.

Next, the survey images are trimmed if needed, linearity corrected with IRLINCOR, divided by exposure time, fixed with the bad pixel mask, and divided by the flat field image. Then the entire stack of images within a strip is used to make a sky image for each filter. The images are median combined using "minmax" rejection with approximately half the images thrown out to reject contributions from stars. The resulting sky image is subtracted from each of the individual frames, and a constant is added back to maintain an appropriate sky level in the reduced image. These images are then assembled into a strip approximately one degree long by cross-correlation of the overlapping regions on each frame. Finally, we derive astrometric solutions for each of our strips by comparing the 248 filter images to the 2MASS catalog images from IPAC and the IRAF task CCMAP.

\subsection{Photometry}

Each frame is analyzed with DoPhot (Schechter et al. 1993). DoPhot identifies, classifies, and performs photometry on objects in an image. It makes successive passes over the image, subtracting the objects and searching in the next pass for fainter objects. The model parameters are found iteratively in the image itself, with reasonable first guesses supplied by the user in parameter files.

\subsection{Apparent magnitude comparison}

Our program has no strict requirement for calibrated photometry since emission-line stars are found through continuum independent line indices. Furthermore, much of the data was taken in non-photometric conditions. However, it is of interest to have an order-of-magnitude calibration in order to help assess how deep the images typically go. We have done a comparison between a typical image section of one of our image 


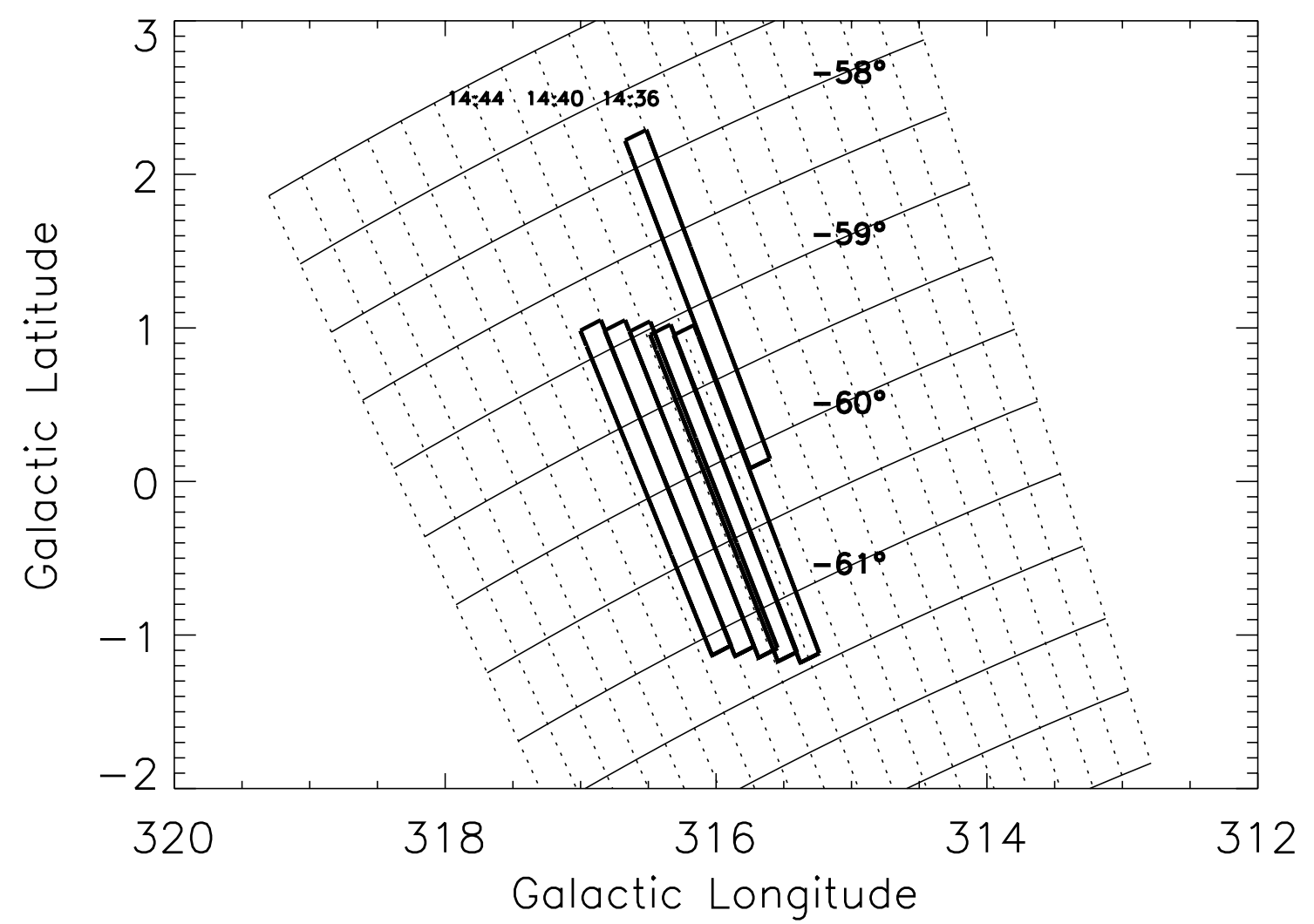

Fig. 4. Same as Fig. 2, but for the 2000 data.

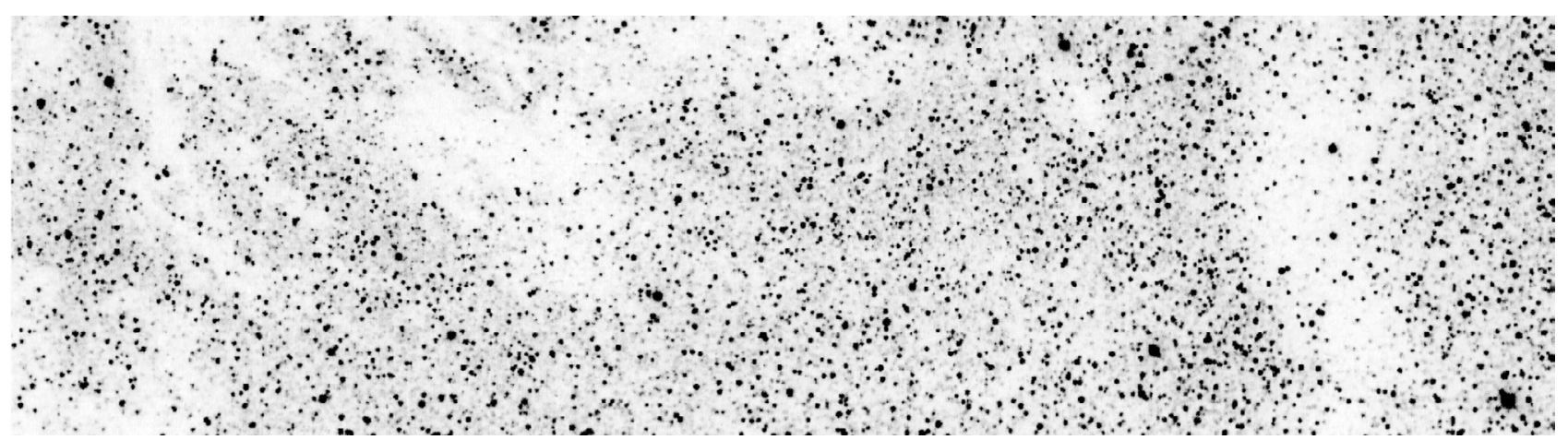

Fig. 5. This is a sample of our data from 1996. The image shown here was taken with the $2.248 \mu \mathrm{m}$ filter. The center coordinates are 17:46:35.7, $-28: 42: 35 \mathrm{~J} 2000$. The $y$ dimension spans $5^{\prime}$ and the $x$ dimension spans $\sim 19^{\prime}$. South is up and east is to the left. Large scale variations in stellar density are apparent and caused by intervening dark clouds.

strips and the 2MASS survey images. We compared our instrumental magnitude for the $2.06 \mu \mathrm{m}$ filter, which is the least sensitive of all our filters due to the combination of transmission efficiency and filter width. 2MASS magnitudes were obtained from the Infrared Science Archive (IRSA) through the GATOR query page. Our comparison shows that an instrumental magnitude of -3 corresponds roughly to an apparent $K$ magnitude of 12 for this strip.

\subsection{Completeness}

To test our sensitivity to emission line stars, we created grids of fake stars with continuum magnitudes ranging from -2.0 to -7.0 in instrumental continuum magnitude, and emission line magnitudes ranging from 0.1 to 1.0 magnitudes brighter than the continuum. That is, we created grids where 100 stars have continuum $(2.03,2.14$, and $2.248 \mu \mathrm{m})$ magnitudes of -2.0 , and line $(2.062,2.077,2.166,2.191 \mu \mathrm{m})$ magnitudes of $-2.1,100$ with the same continuum magnitudes, and line magnitudes of -2.2, and so on. At each step in continuum magnitude, we added 1000 stars with varying line magnitude.

The psf for each filter image was constructed using several bright and relatively well-isolated stars in the image and the IRAF tasks "pstselect" and "psf". Images were then created using "mkobjects". These images were analyzed in exactly the same way as a real image. In Fig. 6 we plot the fraction recovered at increasing brightness of the continuum magnitude. There is a dependence on overall brightness, but no significant 

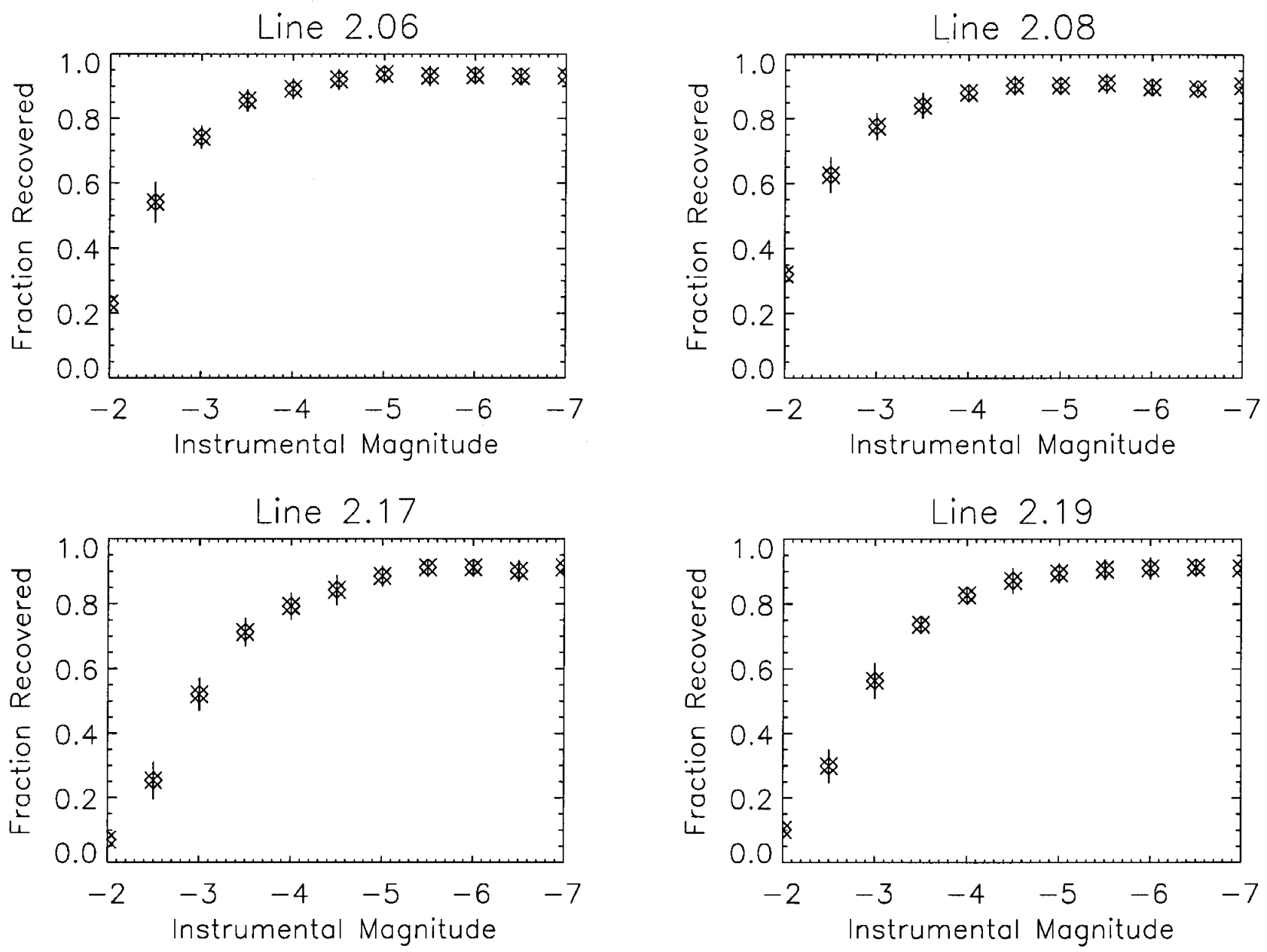

Fig. 6. The fraction of stars recovered in artificial star experiments for the four line images and its dependence on the continuum magnitude of the star. See text.

dependence on emission line strength. This is similar to what one would expect for a broad-band color.

The survey is limited by crowding (especially in the GC region) and the relatively coarse angular resolution element provided by the $1.5 \mathrm{~m}$ telescope. Given the order-of-magnitude comparison made above with the comparison to 2MASS, th $2.06 \mu \mathrm{m}$ filter the data are $\geq 75 \%$ complete at $K=12 \mathrm{mag}$. However, the photometric accuracy decreases at faint magnitudes. This is shown in Fig. 7 for the same stars that are plotted in Fig. 6. The line shown here is the $2.062 \mu \mathrm{m}$ line, but all lines $(2.077,2.166$, and $2.191 \mu \mathrm{m})$ show very similar behavior.

\subsection{Candidate selection}

In this section we detail our candidate selection procedure. As a first step, the object coordinates must be transformed to a single system. We have arbitrarily chosen to use the longest wavelength filter image as our template. Thus, we scale and offset the coordinates for objects in the other lists to the $2.248 \mu \mathrm{m}$ object list. This is accomplished with the "transform" package which accompanies DoPhot. Transform uses a triangular search routine to match stars in different images by comparing the relative scales and orientations of stars in groups of three. Hereafter, we refer to the task as "offset".
We look at each emission-line filter separately, meaning we consider sets of three filters each time, the line and its two continuum filters (2.03 and 2.14 for 2.06 and 2.08; 2.14 and 2.248 for 2.17 and 2.19). The raw output of DoPhot contains 10000 to 30000 objects per image, and this output must first be gleaned for stars with good statistics. The first cut is on object type. In the DoPhot output each object is assigned a number indicating the whether it is a star or a galaxy, and how well it thinks it can determine the magnitude. There are three object types we are interested in: $1 \mathrm{~s}$ are single stars with good statistics, $7 \mathrm{~s}$ are single stars with decent statistics, and $3 \mathrm{~s}$ are fit as members of a blend of 2 single stars. During the transformation to $2.248 \mu \mathrm{m}$ coordinates, "offset" keeps track of the object types in both lists, and records it in the output file. We keep only combinations of single stars (such as $11 \mathrm{~s}, 17 \mathrm{~s}, 71 \mathrm{~s}$, or $77 \mathrm{~s}$ ) and doubles (33 s). We throw out stars which have been classified as a member of a double in one image and a single star in another, as the photometry in this case is very unreliable.

After cleaning the lists for object type, we perform a cut on photometric error. The error is calculated simply as the errors of the individual magnitudes added in quadrature. The error cut is a free parameter in our selection routine, but we usually select it to be between 0.1 and 0.3 mag. 

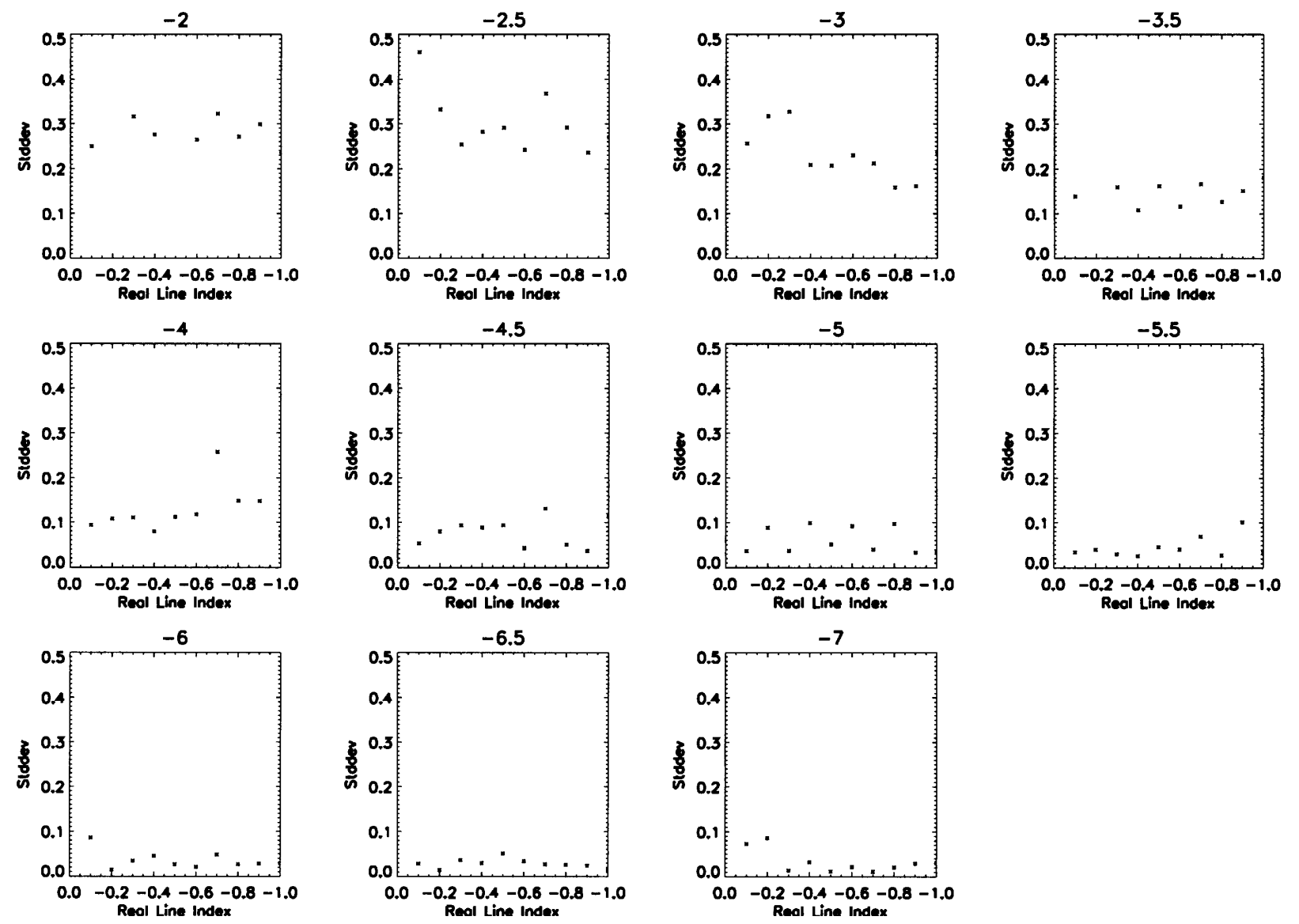

Fig. 7. Standard deviation of the difference between the input color index and the measured line index for the 2.06 line artificial star experiment. The continuum magnitude is shown on the top of each small panel. The error in line index does not depend on line strength, but does depend on continuum magnitude.

We now have three lists of objects, cleaned on the basis of object type and error. The coordinates in these three lists are now matched, and a "line index" is computed as the magnitude difference between the measured value and the expected value derived from a linear interpolation using the two continuum filters magnitudes.

Essentially all stars in the images should have a constant line index (which is not zero due to filter transmission differences and sky transmission variations), that we can subtract to set the mean line index to zero. When the nights were not photometric, systematic line index variations as a function of position (time) along the strip occur. These are relatively straightforward to account for since they produce large scale changes in the line indices with position on the image. In our analysis we subtract these variations by calculating a "mean neighbor index" for each star, using a bin centered on the star, with a size between 50-100 pixels. Smaller bins produce output with less scatter, but the bin must be large enough to contain stars to compute a significant "neighbor index". This mean value is then subtracted from the individual objects.

We also consider the scatter in line index as a function of the short wavelength continuum magnitude. An example is shown in Fig. 8, where the standard deviation in line index is fit as a linear function of short wavelength continuum magnitude. Our final selection is done by considering the line index, the

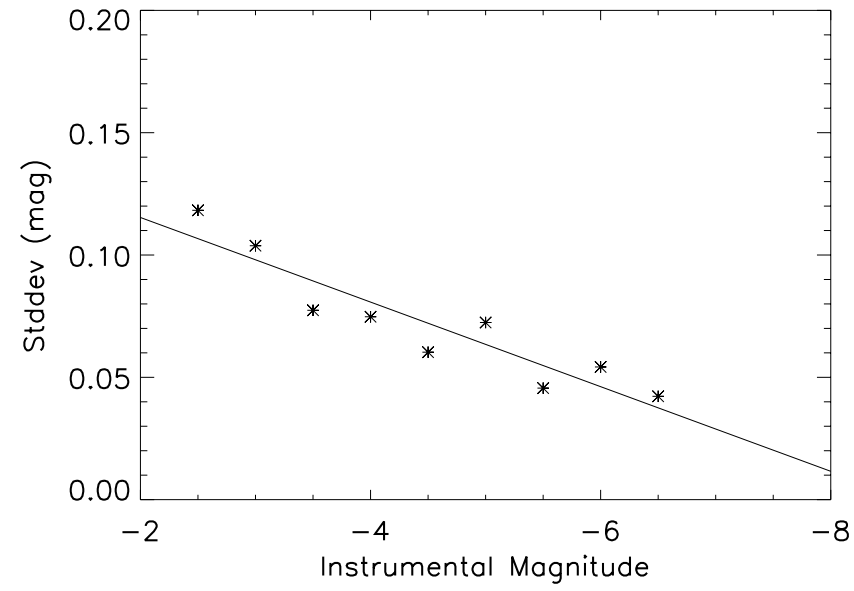

Fig. 8. The scatter as a function of $2.03 \mu \mathrm{m}$ magnitude is fit with a linear function to aid in candidate selection.

error on this index, and the scatter in line index at this magnitude. We employ a criterion similar to the "S" parameter employed by Damineli et al. (1997).

An example of our photometry and candidate selection is shown in Figs. 9 and 10, and candidate emission line objects are overplotted as asterisks. One is a confirmed WR star of late WC subtype (line index $\sim-0.6$, inst. mag. $\sim-3.7$ ) 
(Homeier et al. 2002). In right hand panel of each figure is the the same data plotted, but without correcting for systematic positional variations in line index as described above. One can see that the apparent scatter is larger for the objects in the right-hand panels, and in the case of the 2.08 filter, it affects which objects are chosen as emission-line candidates. In this particular case, a bona-fide WR star would be missed.

\subsection{Image subtraction}

We also use image subtraction as a way to select high priority candidates. This step is only performed for those objects that are selected as photometric candidates. All procedures discussed here are accomplished with standard IRAF routines. An image section is selected within $+/-125$ pixels of a photometric candidate's $x$ position, and a $y$ pixel range from 1 to 250 pixels. First the images are cross-correlated and shifted to a common position. The two continuum filters are averaged and this result is normalized by the mean. The line filter is also normalized by the mean and then divided by the averaged, normalized continuum image. The result is displayed on the screen and examined visually. We have found that this provides a useful and necessary check on the photometric selection. Effects such as bad pixels and residual images are easily seen with this method. We will discuss candidate selection in further detail in the upcoming paper on the spectroscopic follow-up (Homeier et al. 2003, in prep).

\section{Estimating the number of candidates expected}

\subsection{A smooth model}

Following the simple model of Shara et al. (1999), we can estimate the number of candidates we expect to find in the various regions of our survey. We assume a thin, exponential disk, where the stellar density follows a radial exponential law:

$N_{*}=N_{0} \mathrm{e}^{-\left(R-R_{0}\right) / \alpha_{R}}$,

and an exponential dust distribution:

$a_{K}(R)=a_{K, 0} \mathrm{e}^{-\left(R-R_{0}\right) / \alpha_{R}}$,

where $R$ is the Galactocentric distance, $R_{0}$ is the solar Galactocentric distance of $8.0 \mathrm{kpc}, \alpha_{R}=3.0 \mathrm{kpc}$ from Kent et al. (1991). We take $a_{K, 0}$ to be $0.00008 \mathrm{mag} \mathrm{pc}^{-1}$ for consistency with extinction measurements near the Galactic Center (Catchpole et al. 1990). This model predicts a maximum extinction at the Galactic Center of $A_{K} \sim 3.4$.

The WR progenitors, the $\mathrm{O}$ stars, should follow this exponential law, but we must also consider the metallicity dependence for WR formation (Maeder \& Meynet 1994, which modifies $\alpha_{R}$.

$W R / O=0.13 Z / Z_{0}$.

For young stars and emission nebulae, the metallicity variation within the Galactic disk follows an exponential drop-off. Smartt \& Rolleston (1997) found an oxygen gradient in the Galactic disk of:

$$
12+\log (\mathrm{O} / \mathrm{H})=-0.07 \times R_{\mathrm{g}}(\mathrm{kpc})+9.4(\mathrm{dex}) .
$$

This leads us to the final equation for WR stellar density as a function of longitude and distance from the galactic center:

$N_{*}=N_{0, \mathrm{WR}} \mathrm{e}^{-\left(R-R_{0}\right) / \alpha_{\mathrm{WR}}}$,

where $\alpha_{\mathrm{WR}}$ has the value $2022 \mathrm{pc}$ and $N_{0, \mathrm{WR}}=2.2 \mathrm{kpc}^{-2}$ from studies in the local solar neighbourhood (Armandroff \& Massey 1991; Conti \& Vacca 1990; Massey 1996). This relation predicts a total of $\sim 2900$ WR stars in the Galaxy (compared with 2500 in Shara et al. 1999).

We now have an expression for WR density and dust extinction, the next ingredient is absolute $K$ magnitudes for WR stars, which are not well-known. From previous spectral analyses, WC absolute $K$ magnitudes range from -3.8 to -5.7 (Smartt et al. 2001; Dessart et al. 2000; Hillier \& Miller 1999; Crowther et al. 2002). For late-type WCs, which are predominant at high metallicity, absolute $K$ magnitudes range from -3.8 to -5.0 . The absolute $K$ magnitudes of WR134 and WR136, both of WN4-5s type, are -5.47 and -5.64 , respectively (Crowther \& Smith 1996). The Galactic star WR105 (WN9h) has an absolute magnitude of -6.0 derived from its apparent $K$ magnitude, its distance and extinction at $K$ (Churchwell et al. 1992).

Given that WN and WC stars come from a range of initial masses, they should also have a range in absolute magnitude. Additionally, the luminosity of the WNL phase is greater than that of the WC phase while at comparable temperatures. As expected, it appears from observations that WN and WC stars have a range in absolute magnitude, and the WN star population extends to brighter magnitudes. Let us assume a number distribution according to a Salpeter law, that WN stars are distributed between $K-4.0$ and -7.0 , WC stars between -3.0 and -6.0 , and that the $\mathrm{WC} / \mathrm{WN}$ ratio is 1.2 in the inner galaxy (from the empirical relation for $\mathrm{WC} / \mathrm{WN}$ and $\mathrm{O} / \mathrm{H}$ in the Local Group, Massey \& Johnson 1998).

\subsection{Smooth model prediction}

With this model, we can make an estimate of the number of candidates we can expect in our data set. In Fig. 11 we show the number of candidates expected per degree of Galactic longitude as a function of longitude, for varying apparent magnitude limits.

Our images cover $\sim 3$ degrees of longitude, but $\sim 15 \%$ of that is lost by being on the edges of our strips, and 3 strips are of very low signal-to-noise due to clouds. This gives us a total of 2.3 degrees of longitude, covered between \pm 0.5 galactic latitude.

Within that region our model predicts $18 \mathrm{WR}$ stars brighter than $K=11$, and 34 brighter than $K=12$, or 16 additional stars. Our completeness tests indicate that we detect $95 \%$ of emission line stars brighter than $K=11$, and $75 \%$ brighter than $K=12$. According to this, we should detect a total of $28 \mathrm{WR}$ stars in this region. In our second set of data, taken in 2000 at $l=316$, we cover about one degree of longitude between $b \pm 1.0$, and expect 4 WR stars.

So far, we have observed all our high priority candidates in the $\mathrm{GC}$ region. We have not yet observed any candidates 

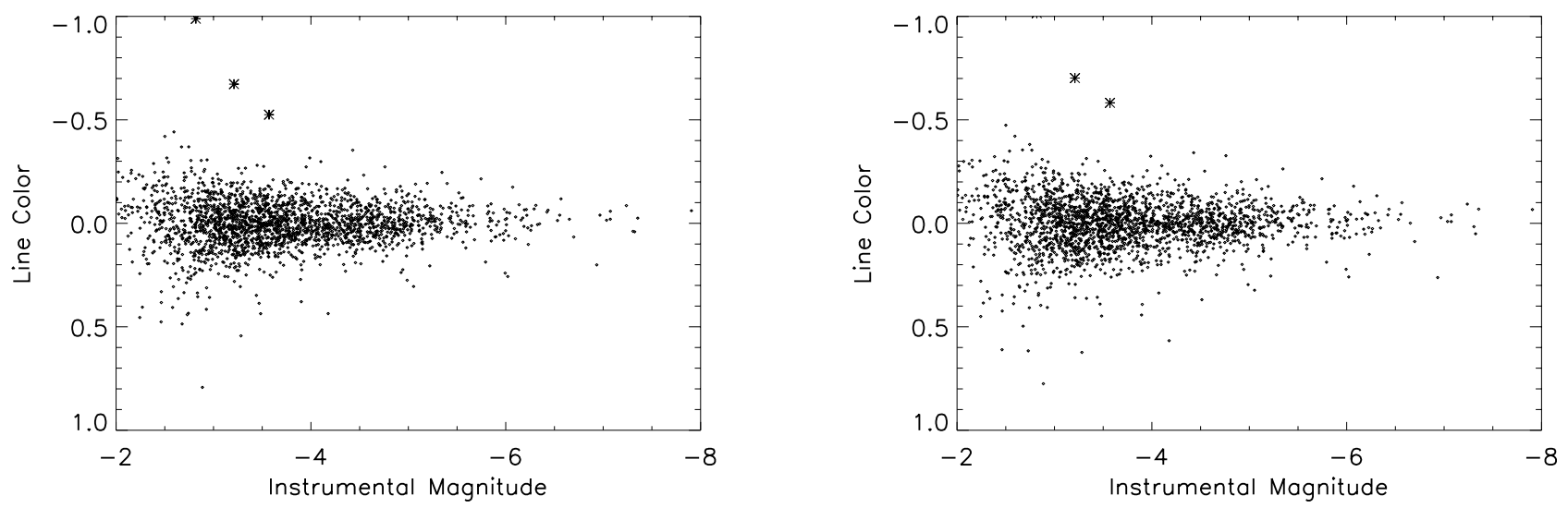

Fig. 9. 2.06 Filter: an example of our photometric data and candidate selection. The stars marked with asterisks are emission line candidates, and one is a confirmed WR star (cindex $\sim-0.6$, mag $\sim-3.7$; Homeier et al. 2002). In the right panel is the same but without correcting for positional line index variations; see text.
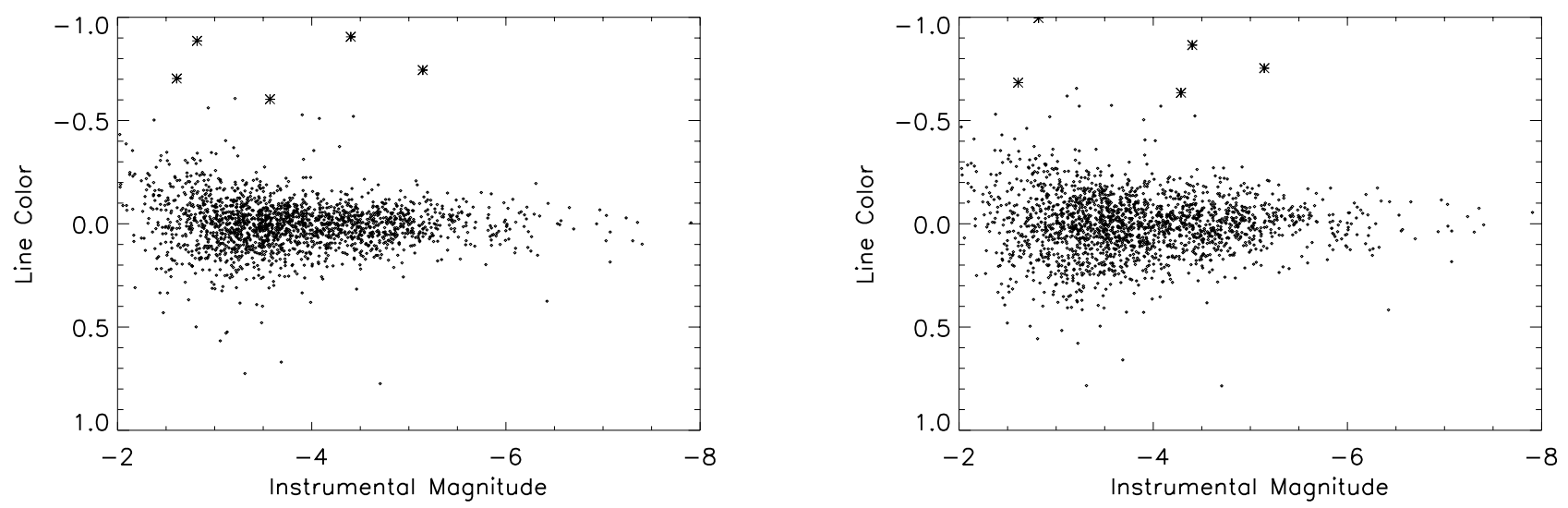

Fig. 10. Same as Fig. 9, but for the $2.08 \mu \mathrm{m}$ filter. The confirmed WR star is located at index $\sim-0.6$, mag $\sim-3.7$.

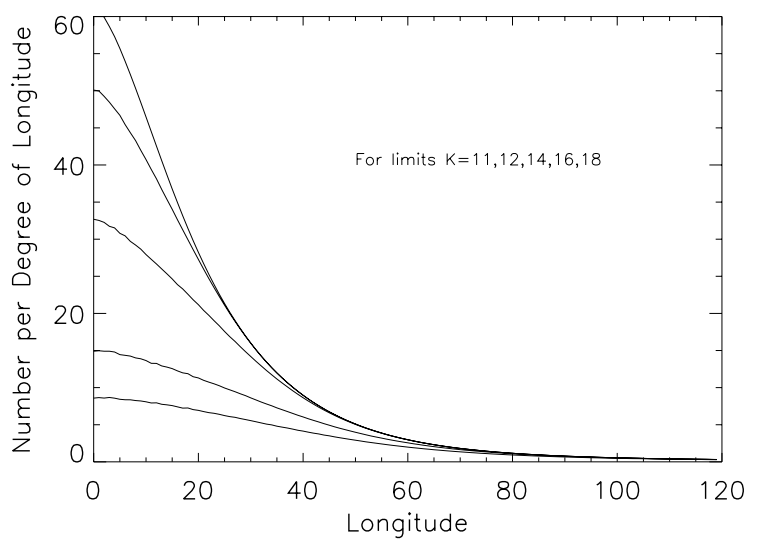

Fig. 11. The number of WR stars expected from our simple model per degree of longitude as a function of Galactic Longitude.

in the Centaurus-Scutum region. In the GC region, we have found four new WR stars. These four stars should be added to those already found in the region through pointed observations. The majority are found in two young clusters, the Arches cluster (Nagata et al. 1995; Cotera et al. 1996), and the Quintuplet cluster (Figer et al. 1999a), though some stars have been found outside clusters as in the present case (Cotera et al. 1999). A full discussion of our detection efficieny and description of the newly discovered WR stars will be given in our upcoming paper on spectroscopic follow-up observations.

Preliminary spectroscopic results are given by Homeier et al. (2002).

\section{Other emission-line objects}

Along with WR stars, we may detect other emission line objects in our survey. As mentioned previously, LBVs are related to WRs, and also have strong emission lines in the infrared. Be and $\mathrm{B}[\mathrm{e}]$ stars have $\mathrm{Br} \gamma$ lines, but much weaker than for WRs or LBVs. Therefore only the strongest emitters may be detected.

Another possibility is WR central stars of planetary nebulae. The stars themselves have very weak lines in this region, but their surrounding nebulae have lines of He I at $2.06 \mu \mathrm{m}$ and B $\gamma$ at $2.166 \mu \mathrm{m}$.

We can also detect very young $\mathrm{O}$ and $\mathrm{B}$ stars still enshrouded in their natal gas and dust. These objects form compact, or ultra-compact H II regions, and later, when they are more revealed, can exhibit emission from circumstellar disks. They will be visible in nebular emission lines of $\mathrm{Br} \gamma$ and He I $2.06 \mu \mathrm{m}$ (Blum \& Damineli 1999b; 
Hanson et al. 2002). Along with the WR stars which we have already detected (see above), we have identified several compact Br $\gamma$ sources. These will be discussed in our following paper detailing the spectroscopic observations of our candidate objects.

\section{Summary}

We have completed a near-infrared survey of approximately three square degrees towards the inner Galaxy including a larger region centered on the Galactic center (GC) and a smaller region towards the Centaurus-Scutum arm. Our survey uses four line emission filters and three continuum filters in the $K$-band. The need for a continuum filter on both the red and blue side of each line is driven by the large and variable extinction toward the inner Galaxy.

We have recently completed spectroscopic follow-up for imaging data taken in 1996-1998 centered on the GC. We discovered four new WR stars. The complete results, including spectra, coordinates, and finding charts, will be published in an upcoming paper (Homeier et al. 2003, in prep). Preliminary spectroscopic results are given by Homeier et al. (2002).

Acknowledgements. The authors would like to thank the referee for a careful reading which improved the paper's final quality. We would also like to acknowledge the continuing excellent support of the CTIO mountain staff. A big thank-you to Ted LaRosa and Michael Nord for providing their $90 \mathrm{~cm}$ radio image, and to P. Eenens for providing his $K$-band WR spectra. N. H. acknowledges and thanks the ESO Studentship Programme and the Wisconsin Space Grant Consortium Graduate Fellowship Program. N. H. would also like to thank the University of Wisconsin Graduate School for partial support. P. S. C. appreciates continuous support from the National Science Foundation. This research has made use of the NASA/IPAC Infrared Science Archive, which is operated by the Jet Propulsion Laboratory, California Institute of Technology, under contract with the National Aeronautics and Space Administration.

\section{References}

Armandroff, T. E., \& Massey, P. 1991, AJ, 102, 927

Blum, R. D., DePoy, D. L., \& Sellgren, K. 1995, ApJ, 441, 603

Blum, R. D., \& Damineli, A. 1999b, ApJ, 512, 237

Blum, R. D., \& Damineli, A. 1999a, in Wolf-Rayet Phenomena in Massive Stars and Starburst Galaxies, ed. K. A. van der Hucht, G. Koenigsberger, \& P. R. J. Eenens (San Francisco: ASP), Proc. IAU, 193, 472

Blum, R. D., Schaerer, D., Pasquali, A., et al. 2001, AJ, 122, 1875

Catchpole, R. M., Whitelock, P. A., \& Glass, I. S. 1990, MNRAS, 247, 479

Churchwell, E., Bieging, J. H., van der Hucht, K. A., et al. 1992, ApJ, 393, 329

Conti, P. S. 1976, in Ministère de l'Éducation Nationale, Fonds National de la Recherche Scientifique and Université de Liège, Colloque International d'Astrophysique 20th, 9, 193
Conti, P. S. 1991, ApJ, 377, 115

Conti, P. S., \& Vacca, W. D. 1990, AJ, 100, 431

Cotera, A. S., Erickson, E. F., Simpson, J. P., et al. 1996, ApJ, 461, 750

Cotera, A. S., Simpson, J. P., Erickson, E. F., et al. 1999, ApJ, 510, 747

Crowther, P. A., \& Smith, L. J. 1996, A\&A, 305541

Crowther, P. A., Dessart, L., Hillier, J., Abbott, J., \& Fullerton, A. 2002, A\&A, 392, 653

Damineli, A., Jablonski, F., de Freitas, L. C., \& de Freitas-Pacheco, J. A. 1997, PASP, 109, 633

Dessart, L., Crowther, P. A., Hillier, J. D., et al. 2000, MNRAS, 315, 407

Figer, D. F., McLean, I. S., \& Morris, M. 1997, ApJ, 486, 420

Figer, D. F., McLean, I. S., \& Morris, M. 1999a, ApJ, 514, 202

Figer, D. F., Kim, S. S., Morris, M., et al. 1999b, ApJ, 525, 750

Hanson, M. M., Luhman, K. L., \& Rieke, G. H. 2002, ApJS, 138, 35

Heckman, T., Robert, C., Leitherer, C., Garnett, D., \& van der Rydt, F. 1998, ApJ, 503, 646

Heckman, T., Sembach, K., Meurer, G., et al. 2001, ApJ, 558, 56

Hillier, J. D., \& Miller, D. L. 1999, ApJ, 519, 354

Homeier, N. L., Blum, R. D., Conti, P. S., Pasquali, A., \& Damineli, A. 2002, in A Massive Star Odyssey, from Main Sequence to Supernova, ed. K. A. van der Hucht, A. Herrero, \& C. Esteban, Proc. IAU Symp., 212 (San Francisco: ASP), in press

Homeier, N. L., Blum, R. D., Pasquali, A., Conti, P. S., \& Damineli, A. 2003, in prep.

Kent, S. M., Dame, T. M., \& Fazio, G. 1991, ApJ, 378, 131

Krabbe, A., Genzel, R., Eckart, A., et al. 1995, ApJ, 447, 95

Langer, N., García-Segura, G., \& Mac Low, M.-M. 1999, ApJ, 520, 49L

LaRosa, T. N., Kassim, N. E., Lazio, T., Joseph, W., \& Hyman, S. D. 2000, AJ, 119, 207

Leitherer, C., Robert, C., \& Drissen, L. 1992, ApJ, 401

Maeder, A., \& Conti, P. S. 1994, ARA\&A, 32, 227

Maeder, A., \& Meynet, G. 1994, A\&A, 287, 803

Maeder, A., \& Meynet, G. 2000, ARAA, 38, 143

Maeder, A., \& Meynet, G. 2001, A\&A, 373, 555

Martin, C. L. 1999, ApJ, 513, 156

Massey, P. M. 1996, in Wolf-Rayet Star in the Framework of Stellar Evolution, ed. J.-M. Vreux, A. Detal, D. Fraipont-Caro, E. Gosset, \& G. Rauw (Liège: Inst. d'Astrophys., Univ. Liège), 361

Massey, P., \& Johnson, O. 1998, ApJ, 505, 793

Meynet, G., \& Maeder, A. 2000, A\&A, 361, 101

Nagata, T., Woodward, C. E., Shure, M., \& Kobayashi, N. 1995, AJ, 109,1676

Oey, M. S., \& Clarke, C. 1997, MNRAS, 289, 570

Oey, M. S., Clarke, C., \& Massey, P. 2001, in Dwarf Galaxies and their Environments, 40th meeting of the Graduiertenkolleg "The Magellanic Clouds and other dwarf galaxies", ed. Klaas S. De Boer, Ralf-Juergen Dettmar, \& Uli Klein (Aachen: Shaker Verlag), 181

Pasquali, A., Langer, N., Schmutz, W., et al. 1997, ApJ, 478, 338

Schechter, P., Mateo, M., \& Saha, A. 1993, PASP, 105, 1342

Shara, M. M., Moffat, A. F. J., Smith, L. F., et al. 1999, AJ, 118, 390

Smartt, S. J., Crowther, P. A., Dufton, P. L., et al. 2001, MNRAS, 325, $257 \mathrm{~S}$

Smartt, S. J., \& Rolleston, W. R. J. 1997, ApJ, 481, L47

Smith, L. J., Nota, A., Pasquali, A., et al. 1998, ApJ, 503, 278 